\title{
Intoxicações por agrotóxicos: Impactos causados pela utilização indiscriminada em
}

\section{comunidades rurais}

\author{
Poisonings by pesticides: Impacts caused by indiscriminate use in rural communities \\ Envenenamientos por plaguicidas: Impactos por uso indiscriminado en comunidades rurales
}

Recebido: 21/06/2021 | Revisado: 29/06/2021 | Aceito: 08/07/2021 | Publicado: 18/07/2021

\author{
Luciana Modesto de Brito \\ ORCID: https://orcid.org/0000-0003-1050-0239 \\ Universidade Federal de Campina Grande, Brasil \\ E-mail: lucianamodesto@hotmail.com \\ Patrício Borges Maracajá \\ ORCID: https://orcid.org/0000-0003-4812-0389 \\ Universidade Federal de Campina Grande, Brasil \\ E-mail: patriciomaracaja@gmail.com \\ Aline Carla de Medeiros \\ ORCID: https://orcid.org/0000-0002-0161-3541 \\ Universidade Federal de Campina Grande, Brasil \\ E-mail: alinecarla.edu@gmail.com \\ Ankilma do Nascimento Andrade Feitosa \\ ORCID: https://orcid.org/0000-0002-4751-2404 \\ Universidade Federal de Campina Grande, Brasil \\ E-mail:ankilmar@hotmail.com \\ Maria Cândida de Almeida Mariz Dantas \\ ORCID: https://orcid.org/0000-0002-6505-7011 \\ Universidade Federal de Campina Grande, Brasil \\ E-mail: candidamariz@yahoo.com.br \\ Antônio Fernandes Filho \\ ORCID: https://orcid.org/0000-0002-3111-7610 \\ Universidade Federal de Campina Grande, Brasil \\ E-mail:antonio.fernandes@professor.ufcg.edu.br
}

\begin{abstract}
Resumo
Objetivo: Analisar os impactos causados pela utilização indiscriminada de agrotóxicos em comunidades rurais. Metodologia: Revisão Sistemática realizada através de buscas, entre fevereiro e maio de 2021, nas bases EMBASE, MEDLINE/PubMed, LILACS, CINAHL e PsycINFO. Resultados: Com a realização da busca foram encontrados 4.593 artigos. Dessa forma, foram removidas 120 duplicatas e 2.644 por não serem dos últimos 10 anos, resultando em 1.829 artigos para a avaliação dos demais critérios de inclusão por meio da leitura dos títulos e resumos. Após avaliação de elegibilidade, 762 artigos apresentaram potencial para inclusão na amostra final do estudo e, dentre esses, 6 foram selecionados para a amostra final após leitura completa. Percebe-se que os impactos são voltados principalmente para a saúde dos agricultores e das pessoas que estão próximas a estes, independentemente de estarem ou não envolvidas diretamente com o manuseio dos agroquímicos. Contudo, nota-se que o principal problema está centrado na falta de educação destes trabalhadores a fim de entenderem acerca do manuseio, proteção e utilização e também de profissionais de saúde local, que não estão aptos a realizar tais atividades Conclusão: Os principais impactos que o uso indiscriminado de agrotóxicos causa para a população de trabalhadores rurais estão centrados nos danos à saúde, principalmente mentais. Ainda, identificou-se que pessoas que estão próximas a estes trabalhadores, como familiares que trabalham ou convivem diretamente ou indiretamente, também estão expostos a sofrem tais danos, evidenciando que a falta de informação é fator preponderante para piora dos casos.
\end{abstract}

Palavras-chave: Praguicidas; Agroquímicos; Assistência à saúde; Assistência integral à saúde; Saúde do trabalhador; Envenenamento.

\footnotetext{
Abstract

Objective: To analyze the impacts caused by the indiscriminate use of pesticides in rural communities. Methodology: Systematic review carried out through searches, between February and May 2021, in EMBASE, MEDLINE/PubMed, LILACS, CINAHL and PsycINFO databases. Results: With the completion of the search, 4,593 articles were found. Thus, 120 duplicates were removed and 2,644 for not being from the last 10 years, resulting in 1,829 articles for the evaluation of the other inclusion criteria by reading the titles and abstracts. After eligibility assessment, 762 articles presented potential for inclusion in the final study sample and, among these, 6 were selected for the final sample after a complete reading. It is noticed that the impacts are mainly aimed at the health of farmers and people who are close
} 
to them, regardless of whether or not they are directly involved in the handling of agrochemicals. However, it is noted that the main problem is centered on the lack of education of these workers in order to understand about the handling, protection and use and also of local health professionals, who are not able to carry out such activities. Conclusion: The main impacts that the indiscriminate use of pesticides causes for the population of rural workers are centered on damage to health, mainly mental. Still, it was identified that people who are close to these workers, such as family members who work or live directly or indirectly, are also exposed to suffering such damages, showing that the lack of information is a major factor for the worsening of cases.

Keywords: Pesticides; Agrochemicals; Delivery of health care; Comprehensive health care; Occupational health; Poisoning.

\section{Resumen}

Objetivo: Analizar los impactos provocados por el uso indiscriminado de plaguicidas en comunidades rurales. Metodología: Revisión sistemática realizada mediante búsquedas, entre febrero y mayo de 2021, en las bases de datos EMBASE, MEDLINE / PubMed, LILACS, CINAHL y PsycINFO. Resultados: Con la finalización de la búsqueda se encontraron 4.593 artículos. Así, se eliminaron 120 duplicados y 2.644 por no ser de los últimos 10 años, resultando 1.829 artículos para la evaluación de los demás criterios de inclusión mediante la lectura de títulos y resúmenes. Después de la evaluación de elegibilidad, 762 artículos presentaron potencial para su inclusión en la muestra final del estudio y, entre estos, 6 fueron seleccionados para la muestra final después de una lectura completa. Se advierte que los impactos están principalmente dirigidos a la salud de los agricultores y personas cercanas a ellos, independientemente de que estén o no directamente involucrados en el manejo de agroquímicos. Sin embargo, se observa que el principal problema se centra en la falta de educación de estos trabajadores para comprender sobre el manejo, protección y uso y también de los profesionales de la salud locales, quienes no son capaces de realizar tales actividades. Conclusión: Los principales impactos que causa uso indiscriminado de plaguicidas en población de trabajadores rurales se centran en los daños a la salud, principalmente mental. Aún así, se identificó que las personas cercanas a estos trabajadores, como los familiares que trabajan o viven directa o indirectamente, también están expuestas a sufrir tales daños, evidenciando que falta de información es un factor importante para el agravamiento de los casos.

Palabras clave: Plaguicidas; Agroquímicos; Atención a la salud; Atención integral de salud; Salud laboral; Envenenamiento.

\section{Introdução}

O Brasil é um dos principais países que mais exporta produtos agropecuários para o mundo, além de estar entre os principais produtores desses produtos no mundo, desempenhando dessa forma uma atividade importante na geração da economia local. Desta forma, para manter uma boa produção são utilizados insumos químicos e sementes transgênicas, como os agrotóxicos e os fertilizantes (Carneiro, 2015).

Por possuir um extenso território e consequentemente uma ampla área de plantio, atualmente o Brasil é o principal consumidor de agrotóxicos no mundo, onde o cultivo dos transgênico, isenção de tributos fiscais, aumento de pragas em lavouras e os créditos agrícolas subsidiados são fatores que contribuem ativamente para o aumento na utilização de produtos químicos nas lavouras (Pignati et al., 2017).

A realização do trabalho rural, onde são utilizados agrotóxicos de forma excessiva para o desenvolvimento agrícola, está relacionado com as condições de saúde do ambiente e dos indivíduos. Dessa forma, os agrotóxicos proporcionam sérios riscos para a saúde, por meio de exposições múltiplas ou únicas durante o manuseio da substância. Essa exposição é responsável por causar morbimortalidade entre todos os países do mundo, principalmente aqueles que ainda encontram-se em processo de desenvolvimento, como a exemplo do Brasil, visto que a situação do país se agrava em decorrência da utilização, cada vez maiores, de quantidades de defensivos agrícolas (Melo et al., 2016).

Visto isso, o uso da proteção de forma inadequada ou até mesmo a falta de utilização dos Equipamentos de Proteção Individual (EPI) correlacionado com a utilização excessiva dos agrotóxicos acarretam em disfunções em vários sistemas do organismo, tais como o sistema gastrointestinal, neurológico, respiratório e na pele, onde o profissional de saúde deve reconhecer esses sintomas correlacionando a exposição ao uso de agrotóxicos aplicando estratégias de diálogo para uma maior aderência a utilização dos EPIs (Tavares et al., 2020). 
É estimado que o número de casos por agrotóxicos ocorra entre um e cinco milhões de pessoas todos os anos, sendo incluídos milhares de casos fatais. A intoxicação por agrotóxicos é um importante problema de saúde pública mundial que afeta principalmente os trabalhadores rurais que possuem baixa escolaridade e que aqueles que não tem acesso a informações de qualidade (Teixeira et al., 2014).

A intoxicação por agrotóxicos pode ser classificada como crônica, subaguda e aguda. Na intoxicação aguda o indivíduo apresenta os sintomas em apenas algumas horas após contato com o agrotóxico. Nesse estágio é típico ocorrer: cefaleia, náuseas, vômitos, irritação da mucosa, dermatite, tontura, cólicas abdominais, dispneia, fraqueza, parestesia, sudorese e salivação. Além disso na forma aguda mais grave o paciente pode sentir pneumonite química, hipotensão, insuficiência respiratória, convulsões, miose, alterações da consciência, coma, choque e até mesmo a morte (Oliveira \& Zambrone, 2006).

Deste modo, o estudo justifica-se pelo fato das intoxicações causadas por agrotóxicos no Brasil está se tornando um sério problema de saúde pública. Onde o aumento no número de diagnósticos, bem como a facilidade no acesso, a crescente utilização e a facilidade no acesso e aquisição do agrotóxico dificulta a vigilância e controle sobre danos que este pode causar a saúde e ao ambiente.

Visto isso, o objetivo do presente estudo é de analisar os impactos causados pela utilização indiscriminada de agrotóxicos em comunidades rurais. Além de averiguar, por meio da literatura, a identificação precoce de uma intoxicação por agrotóxicos, discutir o prognóstico e as consequências de pessoas intoxicadas por agrotóxicos e expor as principais causas de intoxicação entre os trabalhadores rurais.

\section{Metodologia}

Trata-se de uma Revisão Sistemática da literatura, na qual é um tipo de estudo que tem por finalidade identificar, selecionar, avaliar e sintetizar estudos quantitativos, qualitativos e métodos mistos. Visto isso, a revisão sistemática disponibiliza uma síntese do conhecimento já produzido, favorecendo a tomada de decisão precisa entre os profissionais, baseada em evidências (Pluye \& Hong, 2014).

Dessa forma, esse tipo de estudo é construído com base nas seguintes etapas: 1) elaboração da pergunta norteadora; 2) definição dos critérios de inclusão e exclusão; 3) realização de buscas extensiva na literatura; 4) identificação dos estudos por meio de avaliação, inicialmente, do título e posteriormente do resumo; 5) seleção dos artigos; 6) avaliação da qualidade dos mesmos; 7) sistematização dos estudos inclusos (Pluye \& Hong, 2014).

Dessa forma, a revisão foi construída com base na pergunta norteadora: quais os impactos causados em comunidades rurais pela utilização indiscriminada de agrotóxicos?

A busca foi realizada entre fevereiro e maio de 2021, nas bases eletrônicas Excerpta Medica dataBASE (EMBASE), Medical Analysis and Retrievel System Online (MEDLINE/PubMed), Literatura Latino-Americana e do Caribe em Ciências da Saúde (LILACS), Cumulative Index to Nursing and Allied Health Literature (CINAHL) e American Psychological Association (PsycINFO). A busca nas bases de dados ocorreu por meio de descritores obtidos no Medical Subject Headings (MeSH), cuja combinação foi mediada pelos operadores booleanos OR e AND, representado no Fluxograma 1. 
Figura 1 - Fluxograma de busca por meio da combinação de descritores.

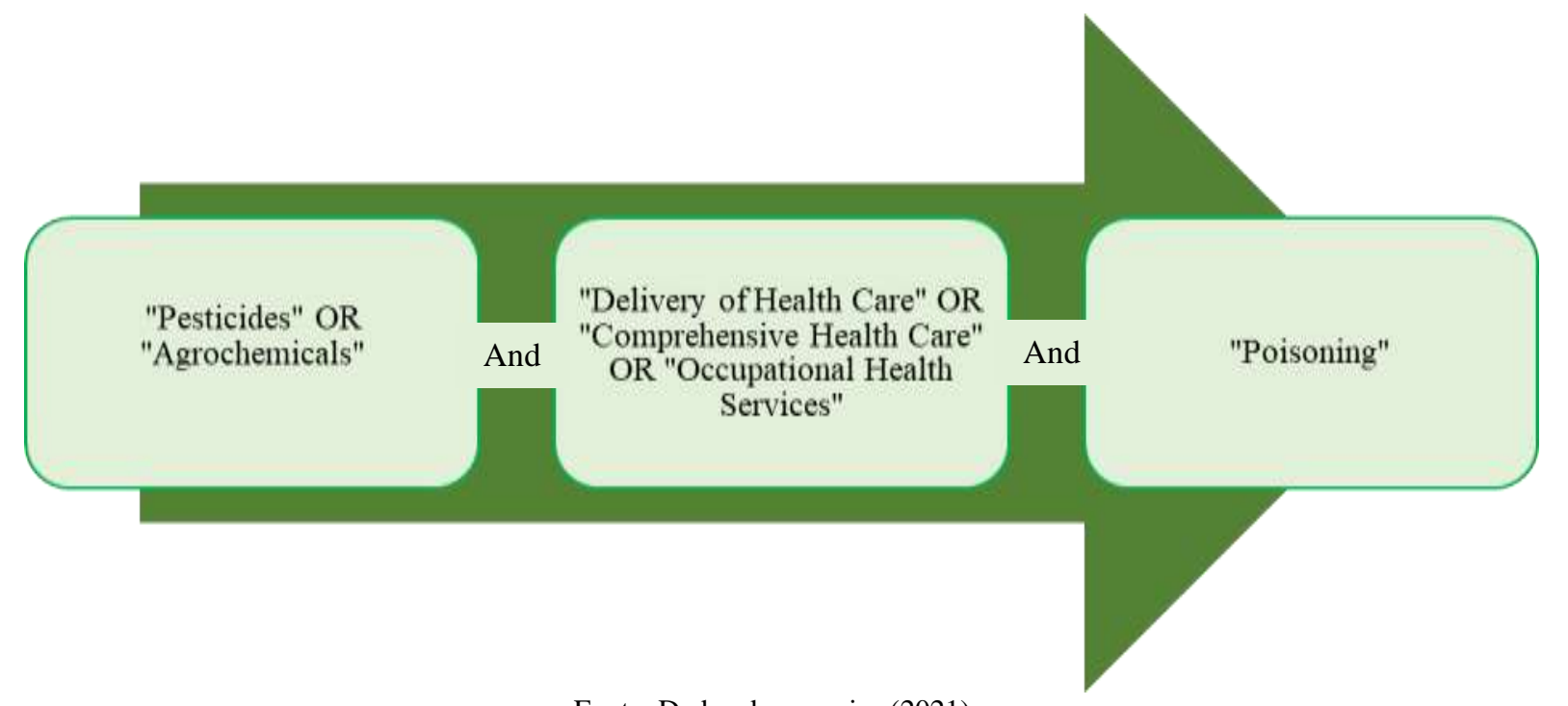

Fonte: Dados da pesquisa (2021).

Para compor os critérios de inclusão da amostra, foram utilizados: estudos com delineamento quantitativo, qualitativo e métodos mistos; artigos publicados em português, inglês e espanhol; gratuitos, disponibilizados na íntegra; publicados nos últimos 10 anos (2000 a 2020). Sendo excluídos os artigos que não abordaram o tema do estudo, sem resumo e sem relato de intoxicação por agrotóxicos.

\section{Resultados}

A busca nas bases de dados gerou 4.593 estudos. A seleção dos artigos foi realizada por meio de exportação dos dados obtidos nas buscas para o gerenciador de referências EndNote versão X8.

Através das utilização do programa, foram excluídos 120 artigos por estarem em duplicata e 2.644 por não terem sido publicados nos últimos 10 anos, restando 1.829 estudos para avaliação dos demais critérios de inclusão, que foram realizados por meio da leitura de títulos e, posteriormente, dos resumos, vale ressaltar que essa etapa foi realizada com a participação de dois revisores independentes. Após avaliação de elegibilidade, 762 artigos apresentaram potencial de inclusão na amostra final e, dentre esses, 6 foram selecionados para a amostra final após a leitura completa (Figura 2). 
Figura 2 - Busca nas bases de dados, adaptação segundo as recomendações PRISMA. Pombal - PB, Brasil, 2021.

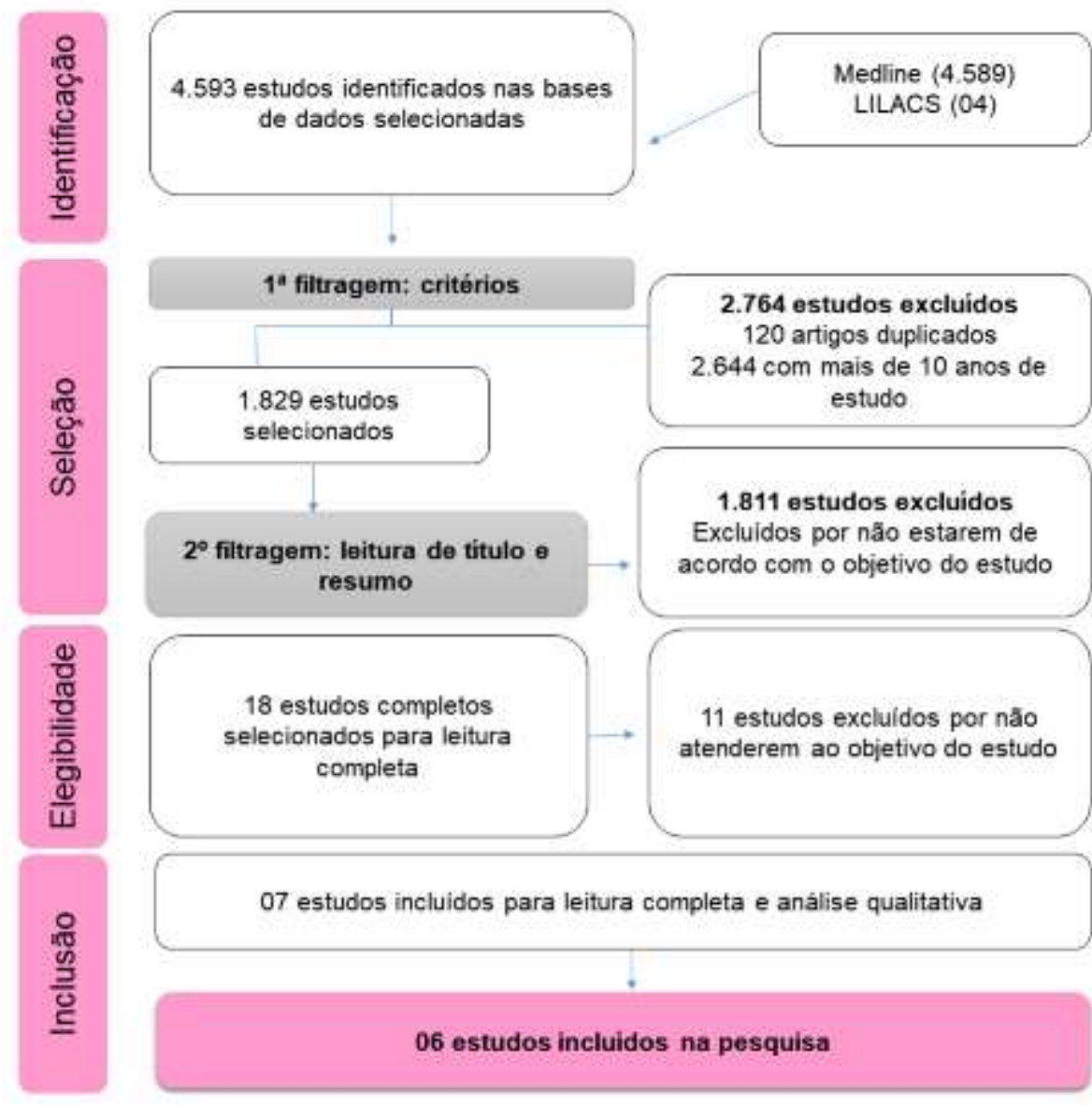

Fonte: Dados da pesquisa (2021).

Dentre os artigos selecionados, $83,3 \%$ eram artigos científicos e apenas $16,7 \%$ trata-se de uma publicação de tese de doutorado. 100\% eram estudos transversais quantitativos, onde 33,3\% foram publicados em 2020,33,3\% publicados em 2014, 16,4\% em 2016 e 16,4\% em 2011 conforme demonstra a Fabela 1.

Tabela 1 - Amostra final de artigos. Pombal/PB, Brasil, 2020.

\begin{tabular}{rcclcl}
\hline Estudo & Autores & Ano & \multicolumn{1}{c}{ Desenho do estudo } & Tipo de produção \\
\hline E1 & Buralli & 2020 & Estudo transversal & Tese de doutorado \\
\hline E2 & Silvério et al. & 2020 & $\begin{array}{l}\text { Estudo transversal, } \\
\text { descritivo e analítico }\end{array}$ & Artigo científico \\
\hline E3 & Cezar-Vaz et al. & 2016 & $\begin{array}{l}\text { Estudo quantitativo, } \\
\text { transversal, observacional e } \\
\text { exploratório }\end{array}$ & Artigo científicico \\
\hline E5 & Lekei Ngowi, \& & 2014 & Estudo transversal & Artigo científico \\
\hline
\end{tabular}

Fonte: Dados da pesquisa (2021).

A Tabela 2 evidencia que os estudos em sua maioria buscaram identificar os impactos causados pelo manuseio dos agrotóxicos na população de trabalhadores agricultores, mas, alguns estudos refletem o acesso a saúde e também o 
envolvimento dos familiares

Tabela 2 - Objetivos e impactos observados na população rural acerca do uso de agrotóxicos. Pombal/PB, Brasil, 2021.

\begin{tabular}{|c|c|c|}
\hline Estudo & Autores & Objetivo \\
\hline E1 & Buralli & $\begin{array}{l}\text { Avaliar os efeitos da exposição aos } \\
\text { pesticidas à saúde de agricultores } \\
\text { familiares em São José de Ubá, } \\
\text { estado do Rio de Janeiro e Analisar } \\
\text { os efeitos respiratórios em crianças } \\
\text { por exposição aos pesticidas. }\end{array}$ \\
\hline E2 & $\begin{array}{c}\text { Silvério et } \\
\text { al. }\end{array}$ & $\begin{array}{l}\text { Avaliar os atributos da atenção } \\
\text { primária à saúde (APS) na } \\
\text { assistência à saúde de trabalhadores } \\
\text { rurais; analisar condições } \\
\text { sociodemográficas, histórico de } \\
\text { intoxicação e internações por } \\
\text { agrotóxicos e uso de equipamentos } \\
\text { de proteção individual; e verificar a } \\
\text { exposição aos praguicidas pela } \\
\text { determinação de bioindicadores. }\end{array}$ \\
\hline E3 & $\begin{array}{c}\text { Cezar-Vaz } \\
\text { et al. }\end{array}$ & $\begin{array}{l}\text { Aplicar uma abordagem } \\
\text { socioambiental na relação entre } \\
\text { saúde humana e trabalho rural por } \\
\text { meio da verificação de } \\
\text { nexo/associação entre distúrbios de } \\
\text { saúde e uso de agrotóxicos. }\end{array}$ \\
\hline
\end{tabular}

E4 Jørs et al. Avaliar o treinamento de pequenos agricultores sobre o manejo de pesticidas e alternativas ecológicas para reduzir os efeitos negativos dos pesticidas.

E5 Lekei Descrever o perfil de exposição dos Ngowi, \& agricultores a pesticidas, London conhecimento sobre os perigos dos pesticidas, experiência de envenenamento anterior, práticas perigosas que podem levar à intoxicação aguda por pesticidas e a extensão em que é relatada

E6 Magauzi
Avaliar os efeitos dos agroquímicos sobre a saúde em trabalhadores agrícolas em fazendas comerciais do distrito de Kwekwe (Zimbábue), em 2006.

\section{Principais impactos}

Agricultores familiares estão ocupacionalmente e ambientalmente expostos aos pesticidas em diversas situações que vão desde a sua vida na infância até a vida enquanto idoso.

Alguns fatores o tornam mais complexos, como: viver próximo de áreas agrícolas, acompanhar seus pais nas áreas de cultivo, começar a ajudar/trabalhar mais cedo na agricultura, realizar misturas de pesticidas, não utilizar EPI'S, hábitos ruins de higiene, realizar uso doméstico dos pesticidas, contato com roupas e equipamentos contaminados, etc.

Os familiares avaliados apresentaram efeitos negativos a saúde associados a esta exposição, trazendo principalmente prejuízos respiratórios demonstrados através dos sinais: rinite, tosse, alergia nasal, aperto no peito, falta de ar, etc. Os trabalhadores rurais são amplamente afetados pela intoxicação advinda dos pesticidas. Com isso, por muitas vezes, possuem como único recurso a atenção primária, mas que não está capacitada para lidar com estas situações, principalmente relacionado aos profissionais.

Os profissionais que atuam em unidades rurais não estão prontos para atender casos de intoxicação, orientações e demais assuntos relacionados a esta temática.

Os principais impactos na saúde humana foram associados a distúrbios mentais $(62,2 \%)$, circulatórios $(49,8 \%)$, dermatológicos $(45 \%)$, respiratórios (41\%) e gástricos $(36,2 \%)$. Os trabalhadores que realização aplicação dos agrotóxicos apresentaram prevalência $90 \%$ maior de alterações dermatológicas quando comparado aos agricultores que não aplicam. Ainda, é possível perceber que independente da aplicação ou não dos pesticidas, a população de agricultores em geral é afetada.

O estudo mostra que o maior impacto para a vida dos agricultores está associada a um baixo nível educacional, citando países como Bolívia e outros de baixa renda.

Contudo, ao realizar treinamentos sobre as formas de proteção, agricultura ecológica e outros assuntos, observou-se redução significativa nas problemáticas associadas a intoxicação, evidenciando a importância da educação nesse tema.

93\% dos agricultores já foram vítimas de envenenamento por pesticidas em seus trabalhos, sendo que destes, $79 \%$ guardavam agrotóxicos em suas casas.

O estudo reforça a importância de ações que não sejam realizadas somente em âmbito pontual e individual, mas sim de maior abrangência para reduzir esta exposição.

Observou-se elevada taxa de intoxicação nos agricultores estudados nas fazendas comerciais, principalmente associados a falta de uso de equipamentos de proteção individual e treinamentos, bem como o manuseio adequado destes agroquímicos. 


\section{Discussão}

Diante dos artigos analisados, percebe-se que os impactos são voltados principalmente para a saúde dos agricultores e das pessoas que estão próximas a estes, independentemente de estarem ou não envolvidas diretamente com o manuseio dos agroquímicos. Contudo, nota-se que o principal problema está centrado na falta de educação destes trabalhadores a fim de entenderem acerca do manuseio, proteção e utilização e também de profissionais de saúde local, que não estão aptos a realizar tais atividades.

Um dos resultados evidenciados mostra que as pessoas próximas aos agricultores também apresentam alto risco de contaminação, o que é mostrado em vários estudos na literatura, onde principalmente as mulheres esposas dos agricultores ou que realizam serviços domésticos, ao lavarem a roupa/EPI contaminados acabam se intoxicando (Abreu \& Alonzo, 2016).

Justifica-se a citação do público feminino em sua maioria como responsável pelo manuseio da higienização destes materiais baseado no estudo de Abreu e Alonzo (2016) onde os homens realizam as atividades em torno da aquisição, transporte, preparo e aplicação; e as mulheres realizam as ações denominadas “do lar".

É na exposição a higienização dos EPI'S que acontece a principal fonte de contaminação destas mulheres ou familiares que realizam essa atividade, tendo em vista que concentra-se principalmente desta forma (Gregolis, Pinto \& Peres, 2012; Abreu \& Alonzo, 2016).

A Associação Nacional de Defesa Vegetal (ANDEF) traz em seus manuais de uso seguro descrevem que é imprescindível a utilização de luvas e aventais no processo de higienização; entretanto, as mulheres relatam em pesquisas que se contaminam com o contato direto, caracterizando a água como “água branca”, que é o líquido que possuem os materiais tóxicos (Iwami et al., 2010; Andef, 2003; Andef, 2006).

Ainda, contando a não utilização dos EPI'S para o processo de higienização, em muitas situações observa-se que a ação é realizada no próprio ambiente familiar, ou seja, na casa do agricultor, o que expõe ainda mais os familiares ao risco de intoxicação (Araújo, Nogueira \& Augusto, 2000; Castro \& Confalonieri, 2004).

Outros fatores estão associados como: não utilização de tanque adequado, via de esgoto inadequada, escolhe de produtos incorreta, utilização de máquina de lavar, escovas ou "bater" a roupa junto com as demais e ações que são constantemente observadas neste público (Araújo, Nogueira \& Augusto, 2000; Castro \& Confalonieri, 2004).

Tais fatores geram a reflexão de que estas famílias realizam as atividades de agricultura em um âmbito familiar e pequeno, promovendo essa geração de toxicidade não só para o agricultor, mas para todo o seu arranjo familiar, tendo em vista que este tipo de trabalho deve ser realizado em locais afastados de casas e com estrutura própria para realização. Contudo, esta não é uma realidade vivenciada pela maior parte dos pequenos agricultores, que necessitam deste trabalho para gerar renda e manter o lar (Araújo, Nogueira \& Augusto, 2000; Castro \& Confalonieri, 2004).

Dessa forma, esses fatores remetem ao que seja o maior problema evidenciado nas publicações analisadas nesta revisão: a baixa escolaridade dos agricultores. Diversos outros estudos tratam este perfil como problemático, uma condição que expressa um importante e elevado risco para este público (Bohner, Araújo \& Nishijima, 2013; Cabral, 2012; Detófano et al., 2013; Siqueira et al., 2013; Tofolo et al., 2014; Ubessi et al., 2015).

Especialmente no Brasil, a maioria majoritária dos estudos evidencia este fato, que consequentemente reflete um nível de conhecimento insatisfatório e que por fim produz uma elevada intoxicação entre agricultores e familiares. Esses danos não circundam apenas em âmbito individual, mas também coletivo e ambiental (Barth \& Biazon, 2010; Santana et al., 2016; Buriola \& Oliveira, 2013).

O baixo nível educacional viabiliza a intoxicação, mas que possuem um percurso até que isto aconteça, como por exemplo a dificuldade de leitura de um rótulo e a compreensão e entendimento frente aos treinamentos que são realizados acerca da temática. Os rótulos dos agrotóxicos possuem composições complexas e necessitam de um conhecimento adequado 
para compreensão e utilização correta, o que em muitos casos, nestes trabalhadores, não é viável (Recena \& Caldas, 2008; Buriola \& Oliveira, 2013).

Contudo, embora o nível educacional seja um problema a ser levado em consideração e um fato que diminui a prevenção frente a intoxicação e utilização dos agrotóxicos, salienta-se que este ainda não é principal problema, e que mesmo que haja uma elevação do nível de escolaridade e mais treinamentos para a área, o principal tópico está associado a infraestrutura para a realização desta prática, que em muitos casos é incompatível com o que se é de fato preconizado.

Esse argumento se reafirma, pois, as implicações para a saúde do trabalhador da agricultura são pautadas principalmente no fato supracitado de os mesmos realizarem tais atividades em seus ambientes familiares, principalmente pela condição socioeconômica em que estes estão inserido (Abreu \& Alonzo, 2016).

Dentro deste contexto, destaca-se ainda o acesso aos serviços de saúde, e trazendo a uma realidade encontrada no Brasil, um dos estudos analisados nesta pesquisa mostra que as Unidades de Saúde que atendem a esta população, que em muitos casos é de baixa escolaridade e baixa renda, não estão preparadas para atenderem estas situações (Silvério et al., 2020).

Estudos demonstram que a não somente os serviços de saúde, mas que muitos serviços municipais em todo o mundo não estão preparados e desconhecem as ações imediatas que devem ser tomadas diante de intoxicações por pesticidas. Ainda se acredita que o principal problema para esse déficit seja a não capacitação dos profissionais para assuntos com estes fins (Jacobson et al., 2009; Bortolotto, Mota \& Tovo-Rodrigues, 2018; Santana, Moura \& Nogueira, 2013).

Concernente a isto, além da falta de informação dos profissionais, o baixo nível de escolaridade e a infraestrutura inadequada, residir em zonas rurais pode potencializar ainda mais estes efeitos, pois o acesso ao centro urbano muitas vezes é dificultado, o que pode, por sua vez, aumentar os efeitos fisiológicos negativos da intoxicação pelos agrotóxicos, passando até despercebido em muitos casos (Jacobson et al., 2009; Bortolotto, Mota \& Tovo-Rodrigues, 2018; Santana, Moura \& Nogueira, 2013).

Estes tópicos em conjunto levam a emergente necessidade de implementação de políticas públicas que venham a gerar uma proteção adequada a estes trabalhadores, que em muitos casos possuem impactos elevados em sua saúde física e psicológica, pois, dentre os principais impactos na saúde dos indivíduos, destacam-se os distúrbios mentais, circulatórios, dermatológicos, respiratórios e gástricos. Inicialmente, é importante salientar que as complicações advindas destes sistemas fisiológicos nem sempre são tratáveis em nível de atenção primária a saúde, mas em sua maioria em nível terciário, o que pode interferir negativamente no acesso desta população (Cesar-Vaz et al., 2016).

O estudo de Cesar-Vaz et al., (2016) mostra que as principais consequências são associadas aos distúrbios mentais. Nesse sentido, observa-se que na população em geral (incluindo a população urbana) os índices de transtornos mentais advindos dos agrotóxicos variam de 29\% a 38\% (Gonçalves, Stein \& Kapczinski, 2008; Rocha Júnior et al., 2004) enquanto que na população rural, esses valores tornam-se mais expressivos, sendo de 36\% a 56\% (Costa \& Ludemir, 2005; Costa, Dimenstein \& Leite, 2014; Paffer et al., 2014).

Além da utilização dos agrotóxicos, o desenvolvimento destes transtornos, podem estar associados a maior presença no sexo feminino, que aliam-se não somente ao trabalho na agricultura, mas atividades que são comuns a estas mulheres, como cuidados com os filhos, casa, cônjuge e baixa visibilidade na realização do seu trabalho (Pinho \& Araújo, 2012; Ludermir, 2005).

Outros fatores já citados também podem ser preponderantes no desenvolvimento de transtornos mentais, como a baixa escolaridade, não uso de EPI's, maior número de intoxicações (Andrade, 2020; Machado, 2018; Butinof et al., 2015).

Destaca-se então o maior número de intoxicações, que devido a quantidade repetida de eventos, o agricultor ou pessoas próximas, tendem a desenvolver com maior facilidade sequelas fisiológicas, em especial os transtornos mentais, como a depressão. Esse resultado é comprovado em diversos estudos (Andrade, 2020; Kim, Ko \& Lee, 2012; Wesseling et al., 2013; 
Beseler \& Stallones, 2008).

Esses fatores levam a reflexão de que as atividades pontuais geram esta situação, mas que a exposição as substâncias contidas nos agrotóxicos, geram prejuízos neurológicos que afetam diretamente no desenvolvimento das mesmas, afirmando a hipótese de que esses trabalhadores em sua maioria os pequenos devem apresentar algum tipo de transtorno.

Com isso, esta pesquisa aponta para a discussão de muitas ideias, tais como: investimento no nível educacional dos agricultores para assim se possa melhorar a percepção dos mesmos sobre os processos de aquisição, transporte, manuseio, armazenamento e descarte dos agrotóxicos; formação e capacitação dos profissionais de saúde para atenderam os agricultores não apenas no ato da intoxicação, mas em nível de prevenção, onde, se deve no sentido da intoxicação já instalada realizar os cuidados imediatos necessários, e encaminhar dentro das possibilidades existentes para um serviço de referência, mas principalmente no papel de prevenção, pois assim, gera-se promoção da saúde a não efetivação da intoxicação em si.

Outros pontos como venda dos agrotóxicos, infraestrutura das fazendas e melhores condições de geração de renda também devem ser discutidos, tendo em vista que como citado anteriormente, embora se tenha a emergente ideia de que o nível educacional seja o principal problema, a infraestrutura inadequada, as más condições de trabalho e os aspectos relacionados ao trabalho familiar são fatores ainda mais eloquentes neste processo.

\section{Conclusão}

Os principais impactos que a utilização indiscriminada dos agrotóxicos causam para a população de trabalhadores agrícolas estão centrados nos danos à saúde, principalmente mentais. Ainda, identificou-se que pessoas que estão próximas a estes trabalhadores, como familiares que trabalham ou convivem diretamente ou indiretamente, também estão expostos a sofrem tais danos, evidenciando que a falta de informação é fator preponderante para piora dos casos.

Os resultados reforçam outras publicações observadas na literatura e mostram que embora o nível educacional seja o principal problema, a infraestrutura dos locais de atuação destes agricultores, que por muitas vezes é familiar e pequena, é o fator que mais acompanha a educação, no sentido de prejudicar estes trabalhadores.

Além disso, é indispensável a realização de estudos futuros sobre a utilização indiscriminada de produtos químicos de utilização agrícola entre a população da comunidade rural, tendo em vista que diversos fatores podem influenciar na intoxicação desses trabalhadores. Esses fatores se relacionam com infraestrutura inadequada, más condições de trabalho e acessibilidade aos produtos químicos.

\section{Referências}

Abreu, P. H. B. D., \& Alonzo, H. G. A. (2016). O agricultor familiar e o uso (in) seguro de agrotóxicos no município de Lavras/MG. Revista Brasileira de Saúde Ocupacional, 41.

Andef, Associação Nacional de Defesa Vegetal. (2003). Manual de uso correto de equipamentos de proteção individual. Linea Creativa.

Andef, Associação Nacional de Defesa Vegetal. (2006). . Manual segurança e saúde do aplicador de produtos fitossanitários. Linea Creativa.

Araújo, A. C., Nogueira, D. P., \& Augusto, L. G. (2000). Impacto dos praguicidas na saúde: estudo da cultura de tomate. Revista de Saúde Pública, 34(3), 309-313.

Barth, V. G., \& Biazon, A. C. B. (2010). Complicações decorrentes da intoxicação por organofosforados. SaBios-Revista de Saúde e Biologia, 5(2).

Beseler, C. L., \& Stallones, L. (2008). A cohort study of pesticide poisoning and depression in Colorado farm residents. Annals of epidemiology, 18(10), 768774.

Bohner, T. O. L., Araújo, L. E. B., \& Nishijima, T. (2013). O impacto ambiental do uso de agrotóxicos no meio ambiente e na saúde dos trabalhadores rurais. Revista eletrônica do curso de direito da UFSM, 8, 329-341.

Bortolotto, C. C., Mola, C. L. D., \& Tovo-Rodrigues, L. (2018). Qualidade de vida em adultos de zona rural no Sul do Brasil: estudo de base populacional. Revista de Saúde Pública, 52, 4s. 
Buralli, R. J. (2020). Efeitos à saúde por exposição ambiental e ocupacional aos pesticidas de uso agrícola (Doctoral dissertation, Universidade de São Paulo).

Buriola, A. A., \& Oliveira, M. L. F. Famílias de agricultores convivendo com praguicidas clandestinos no estado do Paraná-Brasil. Cienc. enferm; 19 (1): 37 47.

Butinof, M., Fernandez, R. A., Stimolo, M. I., Lantieri, M. J., Blanco, M., Machado, A. L., \& Díaz, M. D. P. (2015). Pesticide exposure and health conditions of terrestrial pesticide applicators in Córdoba Province, Argentina. Cadernos de saúde pública, 31, 633-646.

Cabral, E. R. D. M. (2012). Exposição aos agrotóxicos: implicações na saúde de trabalhadores agrícolas de uma região de Campinas - SP. Dissertação (Mestrado em Saúde Coletiva) - Departamento de Saúde Coletiva, Faculdade de Ciências Médicas, Universidade Estadual de Campinas, Campinas.

Carneiro, F. F. (2015). Dossiê ABRASCO: um alerta sobre os impactos dos agrotóxicos na saúde. EPSJV/Expressão Popular.

Castro, J. S. M., \& Confalonieri, U. (2005). Uso de agrotóxicos no Município de Cachoeiras de Macacu (RJ). Ciência \& Saúde Coletiva, 10(2), 473-482.

Cezar-Vaz, M. R., Bonow, C. A., Mello, M. C. V. A. D. \& Silva, M. R. S. D. (2016). Abordagem socioambiental na enfermagem: focalizando o trabalho rural e uso de agrotóxicos. Revista Brasileira de Enfermagem, 69(6), 1179-1187.

Costa, A. G. D., \& Ludermir, A. B. (2005). Common mental disorders and social support in a rural community in Zona da Mata, Pernambuco State, Brazil. Cadernos de Saúde Pública, 21(1), 73-79.

Costa, M. D. G. S. G. D., Dimenstein, M. D. B., \& Leite, J. F. (2014). Condições de vida, gênero e saúde mental entre trabalhadoras rurais assentadas. Estudos de psicologia (natal), 19(2), 145-154.

Detófano, D., Teixeira, M. L., Oliveira, L. F. S., \& Fuentefria, A. M. (2013). Evaluation of toxicity risks in farmers exposed to pesticides in an agricultural community in Concórdia, Santa Catarina State, Brazil. Acta Scientiarum. Health Sciences, 35(1), 111-118.

Gonçalves, D. M., Stein, A. T., \& Kapczinski, F. (2008). Avaliação de desempenho do Self-Reporting Questionnaire como instrumento de rastreamento psiquiátrico: um estudo comparativo com o Structured Clinical Interview for DSM-IV-TR. Cadernos de Saúde Pública, 24, 380-390.

Gregolis, T. B. L., Pinto, W. D. J., \& Peres, F. (2012). Percepção de riscos do uso de agrotóxicos por trabalhadores da agricultura familiar do município de Rio Branco, AC. Revista brasileira de Saúde ocupacional, 37(125), 99-113.

Iwami, A., Ferreira, C. P., Dinnouti, L. A., Bueno, F., Araújo, R. D., Gonsalves, T., \& Santiago, T. (2003). Manual de uso correto e seguro de produtos fitossanitários/agrotóxicos. Linea Creativa.

Jacobson, L. D. S. V., Hacon, S. D. S., Alvarenga, L., Goldstein, R. A., Gums, C., Buss, D. F., \& Leda, L. R. (2009). Comunidade pomerana e uso de agrotóxicos: uma realidade pouco conhecida. Ciência \& Saúde Coletiva, 14(6), 2239-2249.

Jørs, E., Lander, F., Huici, O., Morant, R. C., Gulis, G., \& Konradsen, F. (2014). Do Bolivian small holder farmers improve and retain knowledge to reduce occupational pesticide poisonings after training on Integrated Pest Management? Environmental health, 13(1), 1-9.

Lekei, E. E., Ngowi, A. V., \& London, L. (2014). Farmers' knowledge, practices and injuries associated with pesticide exposure in rural farming villages in Tanzania. BMC public health, 14(1), 1-13.

Ludermir, A. B. (2005). Associação dos transtornos mentais comuns com a informalidade das relações de trabalho. J Bras Psiquiatr, 54(3), 198 -204.

Machado, M. B. (2018). Associação entre exposição a agrotóxicos, depressão e desesperança na população do município de Anahy/pr. Dissertação [Mestrado]. Pós graduação em Biociencias e Saúde. Universidade Estadual do Oeste do Paraná. Cascavel.

Magauzi, R., Mabaera, B., Rusakaniko, S., Chimusoro, A., Ndlovu, N., Tshimanga, M., \& Gombe, N. (2011). Health effects of agrochemicals among farm workers in commercial farms of Kwekwe district, Zimbabwe. Pan African Medical Journal, 9(1).

Melo, W. F., Maracajá, P. B., de Melo, W. F., de Oliveira, T. L. L., Pimenta, T. A., \& de Andrade, A. B. A. (2016). A utilização de agrotóxicos e os riscos a saúde do trabalhador rural. Revista Brasileira de Educação e Saúde, 6(2), 26-30.

Oliveira, M. L. F., \& Zambrone, F. A. D. (2006). Vulnerabilidade e intoxicação por agrotóxicos em agricultores familiares do Paraná. Ciência, Cuidado e Saúde, 5, 099-106.

Paffer, A. T. D., Ferreira, H. D. S., Cabral Júnior, C. R., \& Miranda, C. T. D. (2012). Prevalence of common mental disorders in mothers in the semiarid region of Alagoas and its relationship with nutritional status. São Paulo Medical Journal, 130(2), 84-91.

Pignati, W. A., Lima, F. A. N. D. S., Lara, S. S. D., Correa, M. L. M., Barbosa, J. R., Leão, L. H. D. C., \& Pignatti, M. G. (2017). Distribuição espacial do uso de agrotóxicos no Brasil: uma ferramenta para a Vigilância em Saúde. Ciência \& Saúde Coletiva, 22, 3281-3293.

Pinho, P. D. S., \& Araújo, T. M. D. (2012). Associação entre sobrecarga doméstica e transtornos mentais comuns em mulheres. Revista Brasileira de Epidemiologia, 15(3), 560-572.

Recena, M. C. P., \& Caldas, E. D. (2008). Percepção de risco, atitudes e práticas no uso de agrotóxicos entre agricultores de Culturama, MS. Revista de Saúde Pública, 42(2), 294-301.

Rocha Júnior, D. S., Botelho, J. O. B., Del Fiol, F. S., \& Oshima-Franco, Y. (2004). Síndromes neurológicas induzidas por praguicidas organofosforados e a relação com o suicídio. Saúde em revista, 6 .

Santana, C. M., Costa, A. R. D., Nunes, R. M. P., Nunes, N. M. F., Peron, A. P., Melo-Cavalcante, A. A. D. C., \& Ferreira, P. M. P. (2016). Exposição ocupacional de trabalhadores rurais a agrotóxicos. Cadernos Saúde Coletiva, 24(3), 301-307. 
Research, Society and Development, v. 10, n. 8, e56710817418, 2021

(CC BY 4.0) | ISSN 2525-3409 | DOI: http://dx.doi.org/10.33448/rsd-v10i8.17418

Santana, V. S., Moura, M. C. P., \& Nogueira, F. F. (2013). Mortalidade por intoxicação ocupacional relacionada a agrotóxicos, 2000-2009, Brasil. Revista de Saúde Pública, 47, 598-606.

Siqueira, D. F., de Moura, R. M., Laurentino, G. E. C., de Araújo, A. J., \& Cruz, S. L. (2013). Análise da exposição de trabalhadores rurais a agrotóxicos. Revista Brasileira em Promoção da Saúde, 26(2), 182-191.

Tavares, D. C. G., Shinoda, D. T., da Costa Moreira, S. S., \& da Cruz Fernandes, A. (2020). Utilização de agrotóxicos no Brasil e sua correlação com intoxicações. Sistemas \& Gestão, 15(1), 2-10.

Teixeira, J. R. B., Ferraz, C. E. D. O., Couto Filho, J. C. F., Nery, A. A., \& Casotti, C. A. (2014). Intoxicações por agrotóxicos de uso agrícola em estados do Nordeste brasileiro, 1999-2009. Epidemiologia e Serviços de Saúde, 23, 497-508.

Tofolo, C., Fuentefria, A. M., Farias, F. M., Machado, M. M., \& Oliveira, L. F. S. (2014). Contributing factors for farm workers' exposure to pesticides in the west of the state of Santa Catarina, Brazil. Acta Scientiarum. Health Sciences, 36(2), 153-159.

Ubessi, L. D., Ubessi, C., Kirchner, R. M., Jardim, V. M. D. R., \& Stumm, E. M. F. (2015). Uso de equipamentos de proteção por agricultores que utilizam agrotóxicos na relação com problemas de saúde. Revista de Enfermagem UFPE On Line, Recife, 9(4), 7230-7238.

Wesseling, C., de Joode, B. V. W., Keifer, M., London, L., Mergler, D., \& Stallones, L. (2010). Symptoms of psychological distress and suicidal ideation among banana workers with a history of poisoning by organophosphate or $\mathrm{n}$-methyl carbamate pesticides. Occupational and environmental medicine, 67(11), 778-784. 